\title{
Chiral separation in microflows
}

\author{
Marcin Kostur, Michael Schindler, Peter Talkner, Peter Hänggi \\ Universität Augsburg, Institut für Physik, Universitätsstrasse 1, D-86135 Augsburg, Germany
}

(Dated: July 28, 2021)

\begin{abstract}
Molecules that only differ by their chirality, so called enantiomers, often possess different properties with respect to their biological function. Therefore, the separation of enantiomers presents a prominent challenge in molecular biology and belongs to the "Holy Grail" of organic chemistry. We suggest a new separation technique for chiral molecules that is based on the transport properties in a microfluidic flow with spatially variable vorticity. Because of their size the thermal fluctuating motion of the molecules must be taken into account. These fluctuations play a decisive role in the proposed separation mechanism.
\end{abstract}

PACS numbers: 47.85.Np, 05.60.Cd, 05.10.Gg, 05.40.Jc

The main established methods of enantiomer separation are gas or liquid chromatography and capillary electrophoresis 11. In both cases specific chiral filling materials are used to obtain different elusion times of the enantiomers. Moreover, long columns are needed through which the enantiomers are dragged by a large pressure or a high voltage difference. Chemically less specific methods that work with less substance and that do not require high voltages or pressure would be of great advantage.

During the last ten years the development of microfabricated fluid devices has experienced enormous progress 2]. New ideas how to manipulate small amounts of fluids and substances therein have been suggested [3]. Recently, it has been demonstrated that the agitation of fluids by surface acoustic waves on piezoelectric substrates presents a versatile method of manipulating and controlling the flow of small amounts of a fluid [4, 5].

Various separation mechanisms have been proposed. The separation according to size was predicted for particles in a fluid that is periodically pumped through an array of parallel cylindrical pores with ratchet shaped cylinder radii [6, 7] and experimentally corroborated in Ref. [8]. de Gennes showed that a chiral crystal floating on a liquid surface or gliding on a solid surface under the influence of an external force will generally move in a direction that is specific for the chirality of the crystal [9]. For molecules, de Gennes argued thermal fluctuations would destroy this effect [9]. In the present letter we propose a different mechanism that uses the constructive influence of thermal noise in combination with the nonlinear dynamics of molecules in a flow field to attain a separation of opposite chiral partners.

Inertial forces acting on a suspended point-like particle are generally small [10] and can be neglected. Consequently, the particle is advectively transported with the fluid velocity at the particle's actual position. For an incompressible fluid, the particle motion then is volume conserving, and, as a consequence, attractors do not exist 11]. For small particles, diffusion provides another transport mechanism, which tends to level out concentration differences of suspended particles. In the case of a point particle, diffusion in any incompressible flow results in a homogeneous distribution if external forces like gravity or electric fields are absent.

A contrasting picture results for extended particles. The local velocity of a surface point of an extended particle need not coincide with the fluid velocity that one would observe at this point in the absence of the particle. As a consequence, the volume of the state space spanned by the particle's degrees of freedom is no longer conserved by the dynamics, which, as a result may display attractors for stationary flow fields, in spite of the fact that the dynamics is reversible 12]. In general, several attractors will coexist. Which of them is approached after sufficiently long time depends on the initial conditions. If the particle is still small, say with a diameter of $1 \mu \mathrm{m}$ or less, thermal noise at ambient temperature will destroy very weak attractors and populate stronger ones, with weights depending on the stability of the attractors.

In order to illustrate the working principle, we study a model describing the motion of an extended planar object in a two dimensional incompressible stationary flow. This object, or "molecule" as we will call it, differs from its chiral partners only in the sequence of three spherical "atoms" with different friction coefficients. For a chiral molecule the mirror image does not match with the original molecule upon any motion in the plane. It is the dependence of the transport properties on the chirality which is in the focus of this paper.

At short times, the differences between the dynamics of chiral partners possibly are rather small but at long times they will lead to different attractors with different stability properties. With the omnipresence of thermal noise in the fluid these different stability patterns will cause spatial distributions that are different for chiral partners to an extent that they can be used for an effective chiral separation. This is a robust mechanism that also works in three dimensions.

Motion of an extended molecule in a flowing fluid. We consider three atoms with positions $\mathbf{x}_{i}, i=1,2,3$ that are rigidly connected, see Fig. 1 . The force on the $i$ th particle exerted by the fluid moving with velocity $\mathbf{v}(\mathbf{x})$ is assumed to be proportional to the relative velocity of the particle $\dot{\mathbf{x}}_{i}(t)$ with respect to the fluid, i.e., $\mathbf{F}_{i}^{\mathrm{fl}}=\gamma_{i}\left(\mathbf{v}\left(\mathbf{x}_{i}\right)-\dot{\mathbf{x}}_{i}\right)$ where $\gamma_{i}$ denotes the friction coefficient of the $i$ th atom, and $\mathbf{v}(\mathbf{x})$ is the velocity field of 


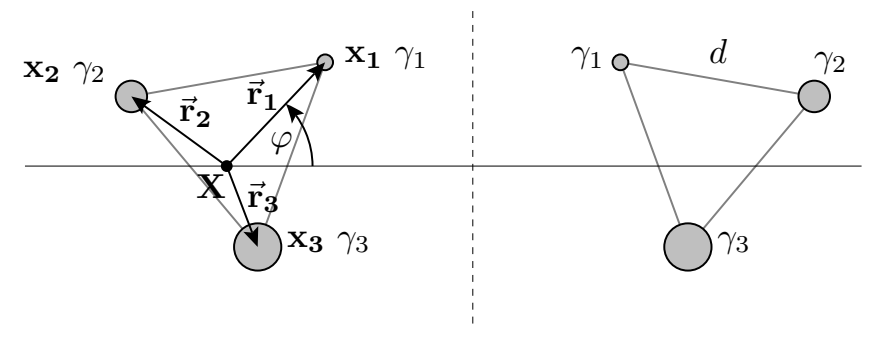

FIG. 1: A chiral molecule consisting of three atoms with different friction coefficients $\gamma_{i}$ on an equilateral triangle at positions $\mathbf{x}_{i}=\mathbf{X}+\mathbf{r}_{i}(\varphi)$. The mirror particle differs only in the sequence of the friction coefficients. The rigid rods (gray lines) of lengths $d$ maintain the structure.

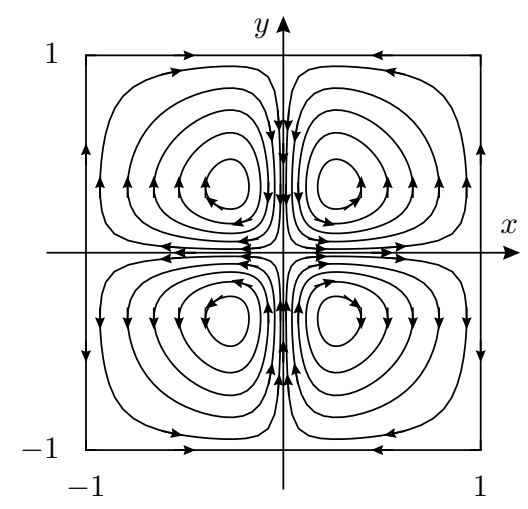

FIG. 2: The streaming pattern given by eq. (7) is shown in one unit cell. The streamlines circulate within adjacent quarters in opposite directions.

the fluid in absence of the atoms. In a fluid at temperature $T$, the frictional forces are always accompanied by random forces, which, for the sake of simplicity, are assumed to be independent Gaussian thermal white noises, $\boldsymbol{\xi}_{i}(t)$, with cartesian components $\xi_{i, \alpha}(t)$, $\alpha=1,2$, satisfying the fluctuation-dissipation relation $\left\langle\xi_{i, \alpha}(t) \xi_{j, \beta}(s)\right\rangle=2 \gamma_{i} k_{B} T \delta_{i, j} \delta_{\alpha, \beta} \delta(t-s)$. We also have to take into account forces $\mathbf{F}_{i, j}$ caused by the $j$ th atom and acting on the $i$ th one that maintain the rigid structure of the molecule. We neglect hydrodynamic interactions between the individual atoms 13. as well as small and very short-lived inertial terms [10]. The particle motion then follows from the balance of forces on each particle

$$
\gamma_{i}\left[\mathbf{v}\left(\mathbf{x}_{i}\right)-\dot{\mathbf{x}}_{i}\right]+\sum_{j \neq i} \mathbf{F}_{i, j}+\boldsymbol{\xi}_{i}(t)=0
$$

The actual degrees of freedom are translations of the molecule as a whole, and rigid rotations. The momentary position of the molecule is conveniently specified by the center of friction $\mathbf{X}=\sum_{i} \tilde{\gamma}_{i} \mathbf{x}_{i}$ where $\tilde{\gamma}_{i}=\gamma_{i} / \sum_{j} \gamma_{j}$ are normalized friction coefficients, and by an angle $\varphi$ fixing the orientation, see Fig. 1 The positions of the atoms can then be recovered as

$$
\mathbf{x}_{i}=\mathbf{X}+\mathbf{r}_{i}(\varphi)=\mathbf{X}+\mathbb{R}(\varphi) \mathbf{r}_{i}^{(0)}
$$

where the vector $\mathbf{r}_{i}(\varphi)$ pointing from $\mathbf{X}$ to the $i$ th atom results from a rotation $\mathbb{R}(\varphi)$ of a reference configuration $\mathbf{r}_{i}^{(0)}$. The center of friction and the orientation then obey coupled Langevin equations reading

$$
\begin{aligned}
\dot{\mathbf{X}} & =\sum_{i} \tilde{\gamma}_{i} \mathbf{v}\left(\mathbf{X}+\mathbf{r}_{i}(\varphi)\right)+\boldsymbol{\xi}_{\mathbf{X}}, \\
\dot{\varphi} & =\sum_{i} \tilde{\gamma}_{i} r_{\gamma}^{-2} \mathbf{r}_{i}^{\prime}(\varphi) \cdot \mathbf{v}\left(\mathbf{X}+\mathbf{r}_{i}(\varphi)\right)+\xi_{\varphi}
\end{aligned}
$$

The dot and the prime denote derivatives with respect to time and angle, respectively. The length $r_{\gamma}=$ $\left(\sum \tilde{\gamma}_{i} \mathbf{r}_{i}^{2}(\varphi)\right)^{1 / 2}$ is independent of $\varphi$ and represents an invariant property of the molecule. The fluctuating forces $\boldsymbol{\xi}_{\mathbf{X}}(t)$ and $\xi_{\varphi}(t)$ are linear combinations of the original ones. They vanish on average, are independent of each other, Gaussian distributed, and therefore characterized by their correlation functions reading

$$
\begin{aligned}
\left\langle\xi_{\mathbf{X} \alpha}(t) \xi_{\mathbf{X} \beta}(s)\right\rangle & =2 D \delta_{\alpha, \beta} \delta(t-s), \\
\left\langle\xi_{\varphi}(t) \xi_{\varphi}(s)\right\rangle & =2 D r_{\gamma}^{-2} \delta(t-s)
\end{aligned}
$$

where the noise strength is given by $D=k_{B} T / \sum_{i} \gamma_{i}$.

In distinct contrast to the two-dimensional velocity field $\mathbf{v}(\mathbf{x})$, the three-dimensional vector field that governs the motion of an extended particle generally has a non-vanishing divergence. It is therefore possible that in the absence of the random forces the long-time dynamics is ruled by one or more attractors which are approached from different initial conditions. The actual structure of the deterministic dynamics will depend on the properties of the molecule and on the considered velocity field $\mathbf{v}(\mathbf{x})$.

The velocity field. The velocity field of an incompressible two-dimensional fluid can always be expressed in terms of a scalar stream function $\Psi(x, y)$. For the sake of definiteness we here choose a periodic stream function

$$
\Psi(x, y)=\frac{V_{0} L_{0} \sin \left(\pi x / L_{0}\right) \sin \left(\pi y / L_{0}\right)}{\left(2-\cos \left(\pi x / L_{0}\right)\right)\left(3-2 \cos \left(\pi y / L_{0}\right)\right)} .
$$

The velocity field follows as $v_{x}(\mathbf{x})=\partial \Psi(\mathbf{x}) / \partial y, v_{y}(\mathbf{x})=$ $-\partial \Psi(\mathbf{x}) / \partial x$. It is the divergence free solution of the Stokes equation [13] for a fluid that is driven by a quadrupolar force density which we do not specify here. Within a unit cell $(x, y \in[-1,1])$ it reproduces the properties of the experimental streaming pattern in Ref. [4]. We use dimensionless variables with $L_{0}=1$ and $V_{0}=\sqrt{15}$. The advective transport follows the lines of constant $\Psi$. Therefore, a unit cell contains four invariant quarters, each containing an eddy. Because the stream function transforms oddly under reflections at the coordinate axes, eddies in two adjacent quarters have an opposite parity according to the sense of rotation. 

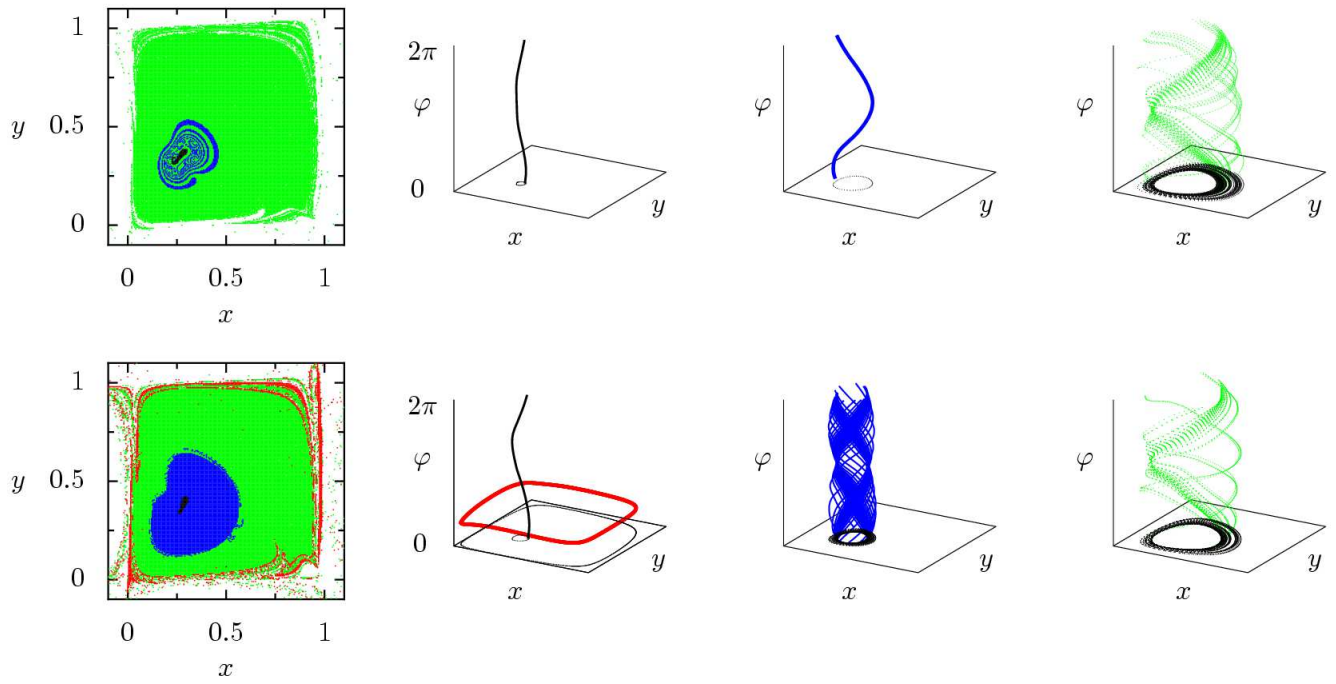

FIG. 3: (color). The deterministic motion of chiral partner molecules (top vs. bottom row) is visualized by the respective domains of attraction (left panels) and the corresponding attractors. The molecule has the shape of an equilateral triangle with side-length $\mathrm{d}=0.2$ and friction coefficients $\left(\gamma_{1}, \gamma_{2}, \gamma_{3}\right)=(1 / 6,1 / 3,1 / 2)$. Its chiral partner has the sequence $\left(\gamma_{1}, \gamma_{2}, \gamma_{3}\right)=$ $(1 / 3,1 / 6,1 / 2)$. Their motions are determined by the deterministic parts of the eqs. (3) 4) with the velocity field (7). The left column shows cuts through the domains of attraction at $\varphi=2$ within the upper right quarter of the unit cell, see Fig. 22 The respective attractors are depicted in the right panels with corresponding colors. Trajectories starting from white regions do not approach the indicated quarter. Note that the domain of attraction of the additional red attractor in the bottom row extends beyond the upper right quarter and consequently attracts trajectories starting outside of this quarter.

Deterministic transport of finite particles. As discussed above, the motion of finite, but still small, particles is qualitatively different from the advective motion of point particles. The deterministic dynamics in the threedimensional state space spanned by the two translational and one orientational degrees of freedom is no longer conservative. It can be partitioned into different domains of attraction. The detailed structure of the attractors and of their corresponding domains of attraction depends on the size of the molecule, on the magnitude of the friction coefficients and also on their sequence and therefore on the chirality of the molecule. for a pair of enantiomers Fig. 3 depicts cuts of the domains of attraction at a fixed orientation $\varphi$ and the corresponding attractors which are confined to one quarter. In the particular case shown in Fig. 3. we found three such attractors for one enantiomer, two of which are period-one attractors, i.e. after one full rotation of $\varphi$ the molecule's center of friction has returned to its initial position. The third attractor is chaotic. In contrast, the respective chiral partner possesses four attractors in this quarter. Three of them are similar to the attractors of the first enantiomer: The chaotic attractor and one of the period-one attractors still exist; the other period-one attractor is replaced by one with winding number 42/43 (42 full rotations of $\varphi$ correspond to 43 revolutions of $\mathbf{X}$ ). The fourth attractor is periodic: Here, the orientation $\varphi$ performs a libration and the translational degrees of freedom move in relatively close distance from the boundary of the considered quarter. This and the chaotic attractors "collect" also points from adjacent quarters, see the left panel of the bottom row in Fig. 3 In contrast, for the chiral partner only a very small set of points from outside the considered quarter is attracted, see the left panel of the top row in Fig. 3. For smaller molecules also more complicated trajectories exist that stay close to the boundaries of several quarters. However, also these trajectories differ for opposite chiral partners and populate preferentially quarters with a parity that is specific for the chirality of the molecule.

Influence of noise. According to their different positions and geometric structures, the attractors of unlike enantiomers have different strengths within a quarter of definite parity, and consequently differ in stability with respect to thermal noise. At sufficiently weak noise almost all molecules settle into the attractor with highest stability, provided the system is given sufficient time to relax into its stationary state. Fig. 4 depicts the resulting unequal distributions of a specific chiral molecule in quarters of different parity. For the transport of a molecule from a quarter with the "wrong" to one with the "fitting" parity the attractors close to the quarters' boundaries, like the red one in Fig. 3. may act as a turnstile.

Because transitions from any less stable to the most stable attractor will become increasingly rare with decreasing noise, the approach to stationarity may proceed slowly. On the other hand, with higher noise levels "wrong" attractors will be populated with increasing probability and therefore the separation quality deteriorates. In Fig. 5 the time to reach the stationary state and the separation performance are compared as they vary with the noise strength, which is quantified by the dimensionless quantity $D / L_{0} V_{0}=k_{B} T / \sum_{i} \gamma_{i} L_{0} V_{0}$. It 


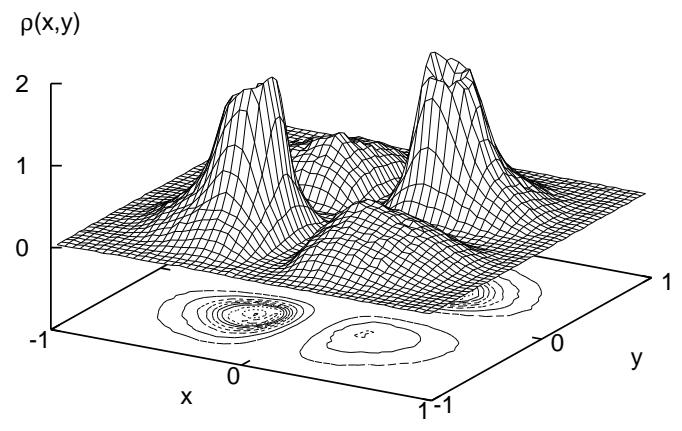

FIG. 4: For a single chiral molecule with size $d=0.2$ the stationary probability density integrated over all orientations $\varphi$ exhibits a pronounced asymmetry with respect to quarters of different parity. The friction coefficients are $\left(\gamma_{1}, \gamma_{2}, \gamma_{3}\right)=(1 / 6,1 / 3,1 / 2)$ and the dimensionless noise strength is $D / L_{0} V_{0}=10^{-3}$.

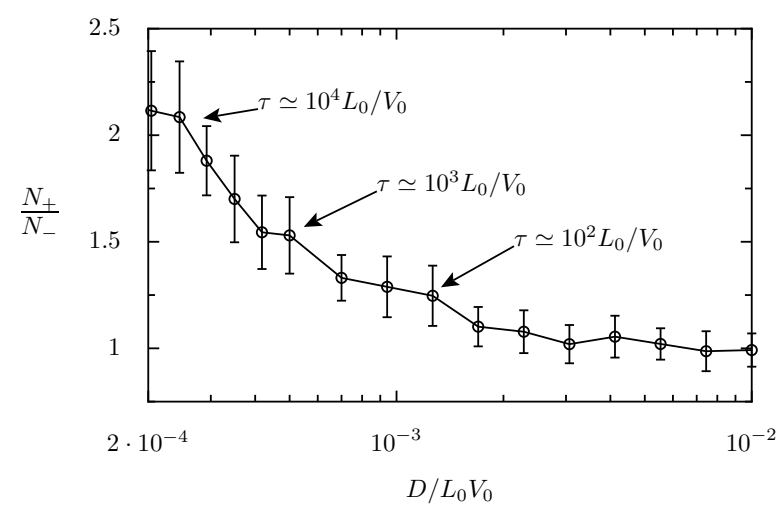

FIG. 5: The selectivity as given by the fraction of numbers of molecules with different chirality $N_{+} / N_{-}$in one quarter decreases with increasing noise strength $D / L_{0} V_{0}$. The time $\tau$ it needs to reach the stationary value increases with decreasing noise strength. For the parameters of the molecule see Fig. 3 The error bars indicate the standard deviation as it results from the numerical simulation.

is therefore not only determined by the temperature but also by the total friction, as well as by the characteristic eddy size and the velocity scale. For example, to achieve a selectivity of $N_{+} / N_{-}=1.5$ within $15 \mathrm{~min}$ in water at room temperature (viscosity $\eta=1 \mathrm{~g} / \mathrm{cm} \mathrm{s}$ ) for this model flow an eddy size of $L_{0} \approx 2 \mu \mathrm{m}$ is required. The size of the molecules then is fixed to $d \approx 0.4 \mu \mathrm{m}$. This eddy size is smaller than what can presently be attained experimentally but it should be realizable in the near future [14]. For this rough estimate we have assumed the validity of the Stokes law. With larger velocity gradients we also expect to improve the separability for smaller objects.

Conclusions. We elucidated the transport properties of a chiral extended object in a two-dimensional model flow in view of efficient enantiomer separation. As a result, we propose to start from a uniform distribution of the racemic mixture and wait until the combined action of the advective transport and of the thermal fluctuations has led to a stationary distribution. In this stationary state, quarters with different parity will contain different amounts of a specific entantiomer.

There are several aspects already in two dimensions which we have not addressed with this investigation. It is nevertheless clear that this model does capture the relevant aspects of symmetry and of the stochastic dynamics of small but extended chiral particles. We are sure that these very aspects do also lead to chiral separation in more realistic models describing e.g. spatially extended atoms, hydrodynamic interactions between them, as well as flexible bonds. Moreover, we are convinced that the same mechanisms also work in a three dimensional helical flow again leading to enantiomer separation. Surface acoustic waves provide a promising tool to produce such flow patterns.

\section{Acknowledgments}

Financial support by the Deutsche Forschungsgemeinschaft via the grants $1517 / 13,1517 / 25$ and SFB 486 B13 is acknowledged.
[1] S. Ahuja, Chromatography and Separation Science, Academic Press, New York, 2002; C. Fujimoto (2002) Anal. Sci. 18, 19(2002).

[2] D. Figeys and D. Pinto, Anal. Chem. 72, 330A (2000).

[3] G. J. M. Bruin Electrophoresis 21, 3931 (2000); J. O. Tegenfeldt, C. Prinz, H. Cao, R. L. Huang, R. H. Austin, S. Y. Chou, E. C. Cox and J. C. Sturm, Anal. Bioanal. Chem. 378, 1678 (2004); H. A. Stone,A. D. Stroock and A. Ajdari, Annu. Rev. Fluid. Mech. 36381 (2004).

[4] Z. Guttenberg, A. Rathgeber, S. Keller, J.O. Rädler, A. Wixforth, M. Kostur, M. Schindler and P. Talkner, Phys. Rev. E 70, 056311 (2004).

[5] Z. Guttenberg, H. Müller, H. Habermüller, A. Geisbauer, J. Pipper, J. Felbel, M. Kielpinski, J. Scriba and A. Wix- forth, Lab on a Chip 5, 308 (2005).

[6] C. Kettner, P. Reimann, P. Hänggi and F. Müller, Phys. Rev. E 61, 312 (2000).

[7] R. D. Astumian and P. Hänggi, Physics Today 55 no.11, 33 (2002); P. Hänggi, F. Marchesoni and F. Nori, Ann. Phys. (Leipzig) 14, 51 (2005).

[8] S. Matthias and F. Müller, Nature 424, 53 (2003).

[9] P. G. de Gennes, Europhys. Lett. 46, 827 (1999).

[10] E. M. Purcell, Am. J. Phys. 45, 3 (1977).

[11] A. J. Lichtenberg, M. A. Liebermann, Regular and Chaotic Dynamics, Springer Verlag, Berlin, 1992.

[12] A. Politi, G. L. Oppo and R. Badii, Phys. Rev. A 33, 4055 (1986).

[13] J. K. G. Dhont, An Introduction to Dynamics of Colloids, 
Elsevier, Amsterdam, 1996.

[14] A. Wixforth and T. Frommelt, private communication. 